Quim. Nova, Vol. 36, No. 4, 544-548, 2013

\title{
TEORES DE TRIGONELINA, ÁCIDO 5-CAFEOILQUÍNICO, CAFEÍNA E MELANOIDINAS EM CAFÉS SOLÚVEIS COMERCIAIS BRASILEIROS
}

\author{
Carolina Tolentino Marcucci e Marta de Toledo Benassi* \\ Departamento de Ciência e Tecnologia de Alimentos, Centro de Ciências Agrárias, Universidade Estadual de Londrina, Rod. Celso \\ Garcia Cid, km 380, 86051-980 Londrina - PR, Brasil \\ Mariana Bortholazzi Almeida e Suzana Lucy Nixdorf \\ Departamento de Química, Centro de Ciências Exatas, Universidade Estadual de Londrina, Rod. Celso Garcia Cid, km 380 , \\ 86051-980 Londrina - PR, Brasil
}

Recebido em 4/6/12; aceito em 14/11/12; publicado na web em 18/2/13

\begin{abstract}
TRIGONELLINE, 5-CAFFEOYLQUINIC ACID, CAFEINE AND MELANOIDINS LEVELS IN COMMERCIAL BRAZILIAN INSTANT COFFEES. Commercial Brazilian regular and decaffeinated instant coffees ( 33 brands) were studied. The levels ranged

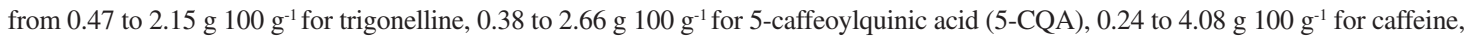
and 0.253 to $0.476(420 \mathrm{~nm})$ for melanoidins. Variations in bioactive compound levels among batches were observed. There was no relationship between the drying process and the composition of the products. In general, Gourmet and decaffeinated coffees had higher trigonelline and 5-CQA but lower caffeine and melanoidin content than regular products.
\end{abstract}

Keywords: bioactive compounds; decaffeinated coffees; HPLC.

\section{INTRODUÇÃO}

Atualmente o Brasil é o maior produtor mundial de café, sendo responsável por $30 \%$ do mercado internacional, e um dos maiores exportadores de café solúvel e café torrado e moído. É também o segundo mercado consumidor, atrás somente dos Estados Unidos. ${ }^{1,2}$ No Brasil o café é a segunda bebida mais consumida entre pessoas acima de 15 anos, atrás somente da água. ${ }^{2}$

As espécies de café mais comercializadas são Coffea arabica e Coffea canephora (robusta) constituindo 70 e $30 \%$ da produção mundial, respectivamente. ${ }^{1,3} \mathrm{O}$ café arábica é um produto com aroma e sabor mais intenso e característico, apresentando maior valor comercial e qualidade sensorial. O café robusta, utilizado em blends com o arábica, tem a finalidade de conferir mais corpo e reduzir a acidez da bebida, ajustando-a a preferência dos consumidores. Por possuir maior teor de sólidos solúveis é amplamente utilizado na indústria de café solúvel, reduzindo o custo do produto. ${ }^{1,4,5}$

Cuidados na colheita, secagem e composição da mistura (espécies, variedades e presença de defeitos) são decisivos para a qualidade dos produtos de café. A fabricação do produto torrado e moído engloba as etapas de composição do blend, processo de torra, moagem, embalagem e armazenamento. ${ }^{6} \mathrm{O}$ café solúvel é o produto resultante da desidratação do extrato aquoso obtido exclusivamente do café torrado, através de métodos físicos, utilizando água como único agente extrator e dependendo do método de desidratação que pode ser classificado em granulado ou aglomerado, pó ou atomizado (spray dried) e liofilizado (freeze dried). ${ }^{7}$

Os cafés arábica e robusta apresentam diferenças no teor de diversos componentes, assim, variações nos blends empregados impactam também na composição e qualidade da bebida. ${ }^{6,8}$ Em grãos verdes, observa-se maior teor de cafeína e ácidos clorogênicos e menor teor de trigonelina no café robusta quando comparado ao café arábica. ${ }^{9}$ Além da matéria-prima, o processo de torra também influencia na composição pela formação e degradação de compostos, sendo que

*e-mail: martatb@uel.br uma maior intensidade de torra aumenta a concentração de produtos de reação de Maillard, como melanoidinas, e reduz os teores de compostos termolábeis, como ácidos clorogênicos e trigonelina. ${ }^{10,11}$ Os processos de extração e, eventualmente, de descafeinação empregados na produção do café solúvel também alteram a composição do produto final. ${ }^{10,12}$

Produtos de café, muitas vezes consumidos pelo seu efeito estimulante e propriedades sensoriais, possuem uma composição química bastante diversificada com presença de componentes responsáveis por efeitos biológicos variados, sendo citados como funcionais (previnem doenças e mantêm a saúde) ou nutracêuticos (propriedades nutricionais e farmacêuticas). ${ }^{13} \mathrm{O}$ efeito benéfico do consumo moderado de café na prevenção de doenças como diabetes tipo II, asma, cirrose alcoólica, Parkinson e Alzheimer, alguns tipos de câncer e na redução no nível de glicose no plasma tem sido descrito. ${ }^{6,13,14} \mathrm{O}$ café destaca-se também pela atividade antioxidante atribuída principalmente à cafeína e trigonelina (comprovada in vivo), compostos fenólicos (in vivo e in vitro) e seus produtos de degradação, e melanoidinas (in vitro).

Dos componentes naturalmente presentes no café, a cafeína, é um alcaloide estável ao processo de torra e o principal psicoativo. A trigonelina, também um alcaloide, é rapidamente degradada durante o processo de torra, dando origem ao ácido nicotínico e compostos aromáticos. ${ }^{6,14,15}$ Os ácidos clorogênicos, termolábeis, possuem propriedades hepatoprotetora, hipoglicêmica e atividade antiviral. ${ }^{6,14,15}$ Entre os compostos formados no processo de torra, as melanoidinas também apresentam atividade antioxidante. ${ }^{13,16}$

Apesar da importância do produto, são bastante restritos os dados de composição de café solúvel. Quando estudados, os autores analisaram poucas amostras, nem sempre utilizam produtos comercias e poucas vezes analisam vários dos componentes bioativos no mesmo trabalho. ${ }^{8,10,17-19}$ Assim, tendo em vista a importância do produto para a economia e dada a existência de poucos dados na literatura, este trabalho teve como objetivo caracterizar cafés solúveis comerciais regulares e descafeinados quanto à composição das principais substâncias bioativas (trigonelina, 5-ACQ, cafeína e melanoidinas). 


\section{PARTE EXPERIMENTAL}

\section{Material}

Foram estudados 33 cafés solúveis produzidos por diferentes processos de secagem (aglomeração, atomização e liofilização), englobando produtos regulares (com cafeína) e descafeinados, totalizando 17 marcas comercializadas no mercado nacional. Os cafés possuíam diferentes denominações no rótulo como: extra forte, forte, tradicional, Gourmet, entre outros. Os cafés Gourmet eram todos desidratados pelo processo de liofilização, os demais eram aglomerados ou em pó. Sempre que possível foram utilizados 3 lotes de cada amostra, totalizando 85 lotes analisados (Tabelas 1 e 2).

\section{Caracterização de umidade e luminosidade}

Os cafés solúveis foram caracterizados quanto à umidade e luminosidade e as análises foram realizadas em triplicata.

Utilizou-se uma balança de umidade equipada com lâmpada halógena (Ohaus MB45, Heuwinkelstrasse, Suíça) com temperatura ajustada em $105^{\circ} \mathrm{C}$ por $7 \mathrm{~min}$. Os valores de umidade foram empregados para cálculo dos teores dos compostos em base seca.

Para determinação da luminosidade $\left(\mathrm{L}^{*}\right)$, as amostras foram acondicionadas em recipiente cilíndrico escuro, e foi utilizado um colorímetro portátil (Konica Minolta CR400, Osaka, Japão), com iluminante D65 e geometria 45/0.

\section{Reagentes}

Os padrões de ácido-5-cafeoilquínico, cafeína e trigonelina com pureza analítica de $\geq 98,0 ; 99,9$ e 99,0\%, respectivamente, foram obtidos da Sigma-Aldrich (Steinheim, Alemanha). A acetonitrila e o ácido acético glacial (grau HPLC), utilizados nas fases móveis, foram adquiridos da J. T. Baker (Phillipsburg, EUA). A água, empregada no preparo da fase móvel e amostras, foi obtida por sistema de purificação e filtração Milli-Q ${ }^{\circledR}$ (Millipore, Molsheim, França). O ácido acético foi filtrado em sistema de filtração a vácuo utilizando membranas de éster de celulose de 0,45 $\mu \mathrm{m}$ (Millipore, São Paulo, Brasil) e para acetonitrila utilizou-se membrana de nylon de $0,22 \mu \mathrm{m}$ (Whatman, Maidstone, Inglaterra).

\section{Determinação de compostos escuros (melanoidinas)}

Os compostos escuros foram estimados adaptando-se a metodologia descrita na literatura. ${ }^{20}$ Os cafés solúveis foram diluídos

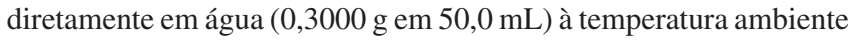
$\left(25^{\circ} \mathrm{C}\right)$ e $0,42 \mu \mathrm{L}$ desse extrato foram adicionados a 4,0 $\mathrm{mL}$ de água, obtendo-se concentração de final de $0,57 \mathrm{mg} \mathrm{mL}^{-1}$. A absorvância das soluções foi medida a $420 \mathrm{~nm}$ em espectrofotômetro UV-VIS Biochrom Libra S22 (Cambridge, Inglaterra). As análises foram realizadas em triplicata. $\mathrm{O}$ valor de absorvância, sugerido por diversos autores como indicativo da formação de compostos escuros na torra de café, foi considerado como estimativa de melanoidinas..$^{21,22}$

\section{Determinação de trigonelina, 5-ACQ e cafeína}

Os cafés solúveis foram diluídos diretamente em solução de ácido acético (5\%), na concentração de $1,0 \mathrm{mg} \mathrm{mL}^{-1}$, e filtrados em membrana de nylon Millex ${ }^{\circledR}$ 0,45 $\mu \mathrm{m}$ (Millipore, São Paulo, Brasil) antes da injeção no cromatógrafo. A análise foi realizada em duplicata.

Foi utilizado um cromatógrafo a líquido de alta eficiência Alliance, modelo e2695 da marca Waters ${ }^{\circledR}$. O sistema cromatográfico consistia de um gerenciador de solventes composto por bomba quaternária e módulo de desgaseificação, amostrador automático com controle de temperatura, forno de colunas e um detector de arranjo de diodos (PDA modelo 2998) controlado pelo software Empower 2 (Waters, Milford, MA, EUA).

As condições cromatográficas foram adaptadas de trabalhos prévios. ${ }^{10,23}$ Empregou-se coluna Spherisorb ${ }^{\circledR}$ ODS 1 (250 x 4,6 mm de diâmetro interno, $5 \mu \mathrm{m}$, Waters, Milford, MA, EUA). Utilizou-se gradiente linear de ácido acético 5\% (A) e acetonitrila (B) na vazão de 1,0 $\mathrm{mL} \mathrm{min}^{-1}$, sendo: 0 a $5 \mathrm{~min}, 3 \%$ de $\mathrm{B} ; 5$ a $10 \mathrm{~min}, 15 \%$ de $\mathrm{B}$; 10 a 20 min, $15 \%$ de B; 20 a 23 min, $3 \%$ de B; 23 a 30 min, $3 \%$ de B. A detecção dos compostos foi realizada no $\lambda$ de máxima absorvância: $260 \mathrm{~nm}$ para trigonelina, $272 \mathrm{~nm}$ para cafeína e $320 \mathrm{~nm}$ para 5-ACQ. O volume de injeção foi de 20,0 mL, com temperatura de coluna controlada em $25^{\circ} \mathrm{C}$.

A identificação foi realizada baseando-se nos tempos de retenção, espectro no UV e fortificação da amostra com padrões. A quantificação foi feita por padronização externa utilizando-se curvas de calibração analíticas construídas a partir das áreas dos picos cromatográficos, empregando-se 12 concentrações de padrão em duplicata (24 pontos). As curvas de calibração mostraram-se lineares na faixa estudada e com alta correlação $\left(R^{2} \geq 0,999\right.$, para $\left.p<0,001\right)$, em intervalos de concentração (faixas dinâmicas de trabalho) de 2,0 a 25,0 $\mathrm{mg} \mathrm{L}^{-1}$ para trigonelina (equação da reta y $=2,2410^{4} \mathrm{x}-1,8810^{3}$ ); 3,0 a 25,0 mg $\mathrm{L}^{-1}$ para 5-ACQ $\left(\mathrm{y}=4,5110^{4} \mathrm{x}-2,3710^{4}\right)$ e 0,2 a $45,0 \mathrm{mg} \mathrm{L}^{-1}$ para cafeína $\left(\mathrm{y}=5,6510^{4} \mathrm{x}+1,1410^{3}\right)$. O método apresentou boa precisão intermediária $\left(\mathrm{CV}_{\text {médio }}=0,54 \%\right.$, para $\mathrm{n}=6 \mathrm{em}$ dias consecutivos $)$.

\section{Análise estatística}

Os resultados foram submetidos à ANOVA, considerando-se a amostra como causa de variação, e teste de médias de Tukey ( $\mathrm{p}$ $\leq 0,05$ ), modificado para número desigual de repetições (Unequal N HSD), utilizando o programa Statistica 7.1. ${ }^{24}$ Os resultados foram comparados dentro de cada categoria (cafés regulares e descafeinados).

\section{RESULTADOS E DISCUSSÃO}

As características (tipo, processamento, umidade e luminosidade) e o perfil de componentes bioativos (trigonelina, 5-ACQ, cafeína e melanoidinas) dos produtos estão apresentados nas Tabelas 1 (cafés regulares) e 2 (descafeinados).

Os produtos apresentaram umidade variando de 2,14 a $6,97 \%$ (média 3,73\%), sendo que apenas 4 dos 85 lotes analisados apresentaram teores acima de $5 \%$, valor máximo permitido pela legislação. ${ }^{25}$ Os produtos apresentaram luminosidade variando de 19,52 a 43,70 (Tabelas 1 e 2).

Um cromatograma típico de cada categoria (regular e descafeinado) pode ser observado na Figura 1.

Além das diferenças já indicadas pelos fabricantes referentes à matéria-prima (Gourmet ou blends), ao processo de secagem (pó, aglomerado ou liofilizado) e processo de descafeinação, observou-se variação de luminosidade ( $L^{*}$ de 19,52 a 43,70) indicando diversidade nas cores de torra e métodos de secagem dos produtos (Tabelas $1 \mathrm{e}$ 2). Para cafés solúveis produzidos com diferentes matérias-primas e processos, já haviam sido relatados valores de $L^{*}$ entre 14 e $33 .{ }^{10}$

Dentre as amostras avaliadas, os cafés regulares apresentaram maior diversidade no tipo, secagem e $\mathrm{L}^{*}$ comparando-se aos observados para cafés descafeinados (Tabelas 1 e 2).

De forma geral, entre os compostos bioativos estudados, os componentes termolábeis (trigonelina e 5-ACQ) se destacaram pela maior variação entre produtos (Tabelas 1 e 2).

Os teores de trigonelina variaram de 0,47 a 2,15 $\mathrm{g} 100 \mathrm{~g}^{-1}$ do 


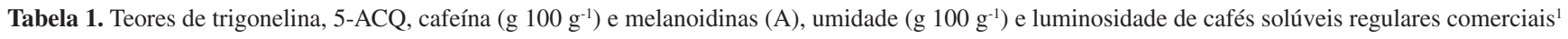

\begin{tabular}{|c|c|c|c|c|c|c|c|}
\hline Produto $^{2}$ & Denominação & Umidade & Luminosidade & Trigonelina $^{3}$ & $5-\mathrm{ACQ}^{3}$ & Cafeína $^{3}$ & Melanoidinas $^{3}$ \\
\hline $\mathrm{A} 1 *$ & $\begin{array}{l}\text { Aglomerado/ } \\
\text { Extraforte }\end{array}$ & $3,96^{a, b, c, d, e, f}(6,82)$ & $23,27^{g, h, i, j}(4,67)$ & $0,95^{\mathrm{d}, \mathrm{f}, \mathrm{g}, \mathrm{h}, \mathrm{i}, \mathrm{j}, \mathrm{k}}(5,92)$ & $0,96^{\mathrm{c}, \mathrm{d}, \mathrm{e}}(12,49)$ & $3,58^{\mathrm{a}, \mathrm{b}, \mathrm{c}, \mathrm{d}, \mathrm{e}, \mathrm{f}}(10,40)$ & $0,36^{\mathrm{a}, \mathrm{b}, \mathrm{c}, \mathrm{d}, \mathrm{e}, \mathrm{f}, \mathrm{g}, \mathrm{h}}(3,86)$ \\
\hline $\mathrm{A} 2 *$ & Aglomerado/Forte & $4,17^{\mathrm{a}, \mathrm{b}, \mathrm{c}}(5,50)$ & $23,11^{g, h, i, j}(3,85)$ & $0,93^{\mathrm{d}, \mathrm{f}, \mathrm{g}, \mathrm{h}, \mathrm{i}, \mathrm{j}, \mathrm{k}}(3,37)$ & $1,14^{\mathrm{c}, \mathrm{d}, \mathrm{e}}(3,21)$ & $3,59^{\mathrm{a}, \mathrm{b}, \mathrm{c}, \mathrm{d}, \mathrm{e}, \mathrm{f}}(0,51)$ & $0,35^{\text {c,d,e,f,g,h,i }}(0,52)$ \\
\hline $\mathrm{A} 3 *$ & Aglomerado & $4,05^{\mathrm{a}, \mathrm{b}, \mathrm{c}, \mathrm{d}, \mathrm{e}}(3,74)$ & $23,90^{\mathrm{f}, \mathrm{g}, \mathrm{h}}(3,79)$ & $0,89^{\mathrm{f}, \mathrm{g}, \mathrm{h}, \mathrm{i}, \mathrm{j}, \mathrm{k}, \mathrm{l}}(1,33)$ & $1,09^{\mathrm{cd, \textrm {d } e \mathrm { e }}}(1,37)$ & $3,67^{\mathrm{a}, \mathrm{b}, \mathrm{c}, \mathrm{d}, \mathrm{e}, \mathrm{f}}(8,54)$ & $0,33^{\mathrm{c}, \mathrm{d}, \mathrm{e}, \mathrm{f}, \mathrm{g}, \mathrm{h,i}}(6,67)$ \\
\hline $\mathrm{A} 4 *$ & Pó & $2,72^{\mathrm{d}, \mathrm{e}, \mathrm{f}}(8,59)$ & $29,47^{\mathrm{c}, \mathrm{d}, \mathrm{e}}(3,68)$ & $0,67^{\mathrm{j}, \mathrm{k}, \mathrm{l}, \mathrm{m}}(5,87)$ & $0,75^{\mathrm{d}, \mathrm{e}}(9,78)$ & $3,62^{a, b, c, d, e, f}(2,88)$ & $0,42^{\mathrm{a}, \mathrm{b}, \mathrm{c}}(5,50)$ \\
\hline $\mathrm{B} 1 *$ & Aglomerado & $2,60^{f}(18,62)$ & $23,32^{\mathrm{g}, \mathrm{h}, \mathrm{ij}}(0,82)$ & $0,80^{\mathrm{g}, \mathrm{h}, \mathrm{j}, \mathrm{j}, \mathrm{k}, \mathrm{lm}}(8,64)$ & $0,87^{\mathrm{c}, \mathrm{d}, \mathrm{e}}(19,21)$ & $3,35^{\mathrm{d}, \mathrm{fg}}(6,49)$ & $0,43^{\mathrm{a}, \mathrm{b}, \mathrm{c}}(2,60)$ \\
\hline $\mathrm{B} 2 *$ & Liofilizado/Gourmet & $3,31^{\mathrm{a}, \mathrm{b}, \mathrm{c}, \mathrm{d}, \mathrm{e}, \mathrm{f}}(12,69)$ & $34,78^{b}(3,43)$ & $1,15^{\mathrm{c}, \mathrm{d}, \mathrm{e}, \mathrm{f}}(5,90)$ & $1,15^{\mathrm{c}, \mathrm{d}, \mathrm{e}}(12,15)$ & $2,69^{\mathrm{h}, \mathrm{ij}}(2,76)$ & $0,34^{\mathrm{c}, \mathrm{d}, \mathrm{e}, \mathrm{f}, \mathrm{g}, \mathrm{h,i}, \mathrm{i}}(6,51)$ \\
\hline B3* & Pó & $2,73^{\mathrm{c}, \mathrm{d}, \mathrm{e}, \mathrm{f}}(6,74)$ & $33,00^{\mathrm{b}, \mathrm{c}}(2,86)$ & $1,17^{\mathrm{c}, \mathrm{d}, \mathrm{e}, \mathrm{f}}(3,23)$ & $2,34^{\mathrm{a}}(2,87)$ & $3,51^{\mathrm{b}, \mathrm{c}, \mathrm{d}, \mathrm{e}, \mathrm{f}}(1,79)$ & $0,31^{\mathrm{d}, \mathrm{e}, \mathrm{f}, \mathrm{g}, \mathrm{h,i}}(5,82)$ \\
\hline $\mathrm{C} 1^{*}$ & Aglomerado & $3,79^{a, b, c, c, d, e, f}(7,28)$ & $19,52^{\mathrm{j}}(2,85)$ & $1,04^{\mathrm{d}, \mathrm{e}, \mathrm{f}, \mathrm{g}, \mathrm{h}}(9,92)$ & $0,98^{\mathrm{c}, \mathrm{d}, \mathrm{e}}(8,10)$ & $3,79^{a, b, c, d, e, f}(5,73)$ & $0,40^{\mathrm{a}, \mathrm{b}, \mathrm{c}, \mathrm{d}}(4,99)$ \\
\hline $\mathrm{C} 2 *$ & Liofilizado/Gourmet & $3,02^{b, c, d, e, f}(6,13)$ & $34,63^{b}(3,74)$ & $1,29^{\mathrm{b}, \mathrm{c}, \mathrm{e}}(10,18)$ & $1,37^{\mathrm{b}, \mathrm{c}, \mathrm{d}}(12,23)$ & $2,62^{\mathrm{i}, \mathrm{j}}(6,60)$ & $0,39^{\mathrm{a}, \mathrm{b}, \mathrm{c}, \mathrm{d}, \mathrm{e}, \mathrm{g}}(6,63)$ \\
\hline $\mathrm{C} 3 *$ & Pó & $4,27^{\mathrm{a}, \mathrm{b}}(1,43)$ & $30,18^{\mathrm{c}, \mathrm{d}}(1,53)$ & $0,90^{f, g, h, i, j, k, 1}(5,62)$ & $1,16^{\mathrm{cd, \textrm {de }}}(3,91)$ & $3,61^{\mathrm{a}, \mathrm{b}, \mathrm{c}, \mathrm{d}, \mathrm{e}, \mathrm{f}}(2,72)$ & $0,29^{\mathrm{f}, \mathrm{g}, \mathrm{h}, \mathrm{i}}(12,92)$ \\
\hline $\mathrm{C} 4 *$ & Aglomerado & $4,16^{\mathrm{a}, \mathrm{b}, \mathrm{c}, \mathrm{d}}(6,01)$ & $20,74^{\mathrm{h}, \mathrm{i}, \mathrm{j}}(1,68)$ & $0,59^{1, \mathrm{~m}}(11,71)$ & $0,59^{\mathrm{e}}(17,11)$ & $3,88^{\mathrm{a}, \mathrm{b}, \mathrm{c}, \mathrm{e}}(2,89)$ & $0,36^{\text {a,c,de,f,g,g, }}(6,19)$ \\
\hline $\mathrm{C} 5^{*}$ & Aglomerado & $2,80^{\mathrm{c}, \mathrm{d}, \mathrm{e}, \mathrm{f}}(34,98)$ & $22,45^{\mathrm{h}, \mathrm{ij}}(10,90)$ & $0,71^{\mathrm{i}, \mathrm{j}, \mathrm{k}, \mathrm{l}, \mathrm{m}}(13,16)$ & $0,75^{\mathrm{d}, \mathrm{e}}(18,04)$ & $3,45^{\mathrm{c}, \mathrm{d}, \mathrm{e}, \mathrm{f}}(3,84)$ & $0,46^{\mathrm{b}}(2,19)$ \\
\hline $\mathrm{D} 1 *$ & Aglomerado & $3,85^{a, b, c, d, e, f}(3,34)$ & $23,79^{\mathrm{g}, \mathrm{h}}(1,93)$ & $0,65^{\mathrm{k}, \mathrm{lm}}(2,09)$ & $0,69^{\mathrm{e}}(6,48)$ & $4,08^{\mathrm{a}}(0,29)$ & $0,39^{\mathrm{a}, \mathrm{b}, \mathrm{c}, \mathrm{d}, \mathrm{e}, \mathrm{g}}(1,80)$ \\
\hline $\mathrm{D} 2 *$ & Pó & $2,67^{\mathrm{e}, \mathrm{f}}(14,82)$ & $27,67^{\mathrm{d}, \mathrm{e}, \mathrm{f}}(5,49)$ & $0,99^{\mathrm{d}, \mathrm{e}, \mathrm{fg}, \mathrm{gh,i.j}}(6,46)$ & $1,36^{\mathrm{b}, \mathrm{c}, \mathrm{d}}(10,88)$ & $3,98^{\mathrm{a}, \mathrm{b}, \mathrm{c}}(2,02)$ & $0,34^{\mathrm{c}, \mathrm{de}, \mathrm{f}, \mathrm{g}, \mathrm{gh,i}}(13,72)$ \\
\hline E1* & Aglomerado & $3,81^{a, b, c, c, d, f, f}(3,56)$ & $22,57^{\mathrm{h}, \mathrm{i}, \mathrm{j}}(4,03)$ & $1,03^{\mathrm{d}, \mathrm{e}, \mathrm{f}, \mathrm{g}, \mathrm{h}}(5,01)$ & $1,46^{\mathrm{b}, \mathrm{c}}(3,13)$ & $3,74^{a, b, c, d, e, f}(3,40)$ & $0,30^{e, f, g, h, i}(6,99)$ \\
\hline $\mathrm{E} 2 * * *$ & Pó & $3,75^{\mathrm{a}, \mathrm{b}, \mathrm{c}, \mathrm{d}, \mathrm{e}, \mathrm{f}}(2,41)$ & $26,94^{\mathrm{de}, \mathrm{f}, \mathrm{g}}(2,11)$ & $0,47^{\mathrm{m}}(0,43)$ & $0,38^{\mathrm{e}}(0,86)$ & $3,52^{\mathrm{a}, \mathrm{b}, \mathrm{c}, \mathrm{d}, \mathrm{e}, \mathrm{f}}(0,13)$ & $0,48^{\mathrm{a}, \mathrm{b}}(2,67)$ \\
\hline $\mathrm{F} 1^{*}$ & Aglomerado & $4,00^{a, b, c, c, e, f, f}(8,26)$ & $20,73^{\mathrm{h}, \mathrm{i,j}}(4,39)$ & $1,60^{\mathrm{b}}(7,49)$ & $2,66^{a}(10,78)$ & $3,89^{\mathrm{a}, \mathrm{b}, \mathrm{c}, \mathrm{e}}(5,88)$ & $0,28^{\mathrm{fh}, \mathrm{i}}(4,57)$ \\
\hline $\mathrm{G} 1 *$ & Pó & $4,40^{\mathrm{a}, \mathrm{b}}(31,69)$ & $20,03^{\mathrm{d}, \mathrm{e}}(2,07)$ & $1,00^{\mathrm{d}, \mathrm{e}, \mathrm{f}, \mathrm{h}, \mathrm{i}}(4,50)$ & $1,45^{\mathrm{b}, \mathrm{c}}(6,30)$ & $3,56^{a, b, c, d, e, f}(1,59)$ & $0,36^{\mathrm{c}, \mathrm{d}, \mathrm{e}, \mathrm{f}, \mathrm{g}, \mathrm{h}}(7,41)$ \\
\hline $\mathrm{H} 1 *$ & Pó & $3,55^{a, b, c, d, e, f}(6,56)$ & $19,85^{\mathrm{i}, \mathrm{j}}(5,71)$ & $0,87^{\mathrm{f}, \mathrm{g}, \mathrm{h}, \mathrm{i}, \mathrm{j}, \mathrm{k}, \mathrm{l}}(19,18)$ & $0,81^{\mathrm{d}, \mathrm{e}}(28,40)$ & $3,88^{\mathrm{a}, \mathrm{b}, \mathrm{c}, \mathrm{e}}(2,18)$ & $0,40^{\mathrm{a}, \mathrm{b}, \mathrm{c}, \mathrm{d}, \mathrm{e}}(2,30)$ \\
\hline $\mathrm{I} 1 *$ & Aglomerado & $4,32^{\mathrm{a}, \mathrm{b}}(8,45)$ & $23,46^{\mathrm{g}, \mathrm{h}, \mathrm{i}}(3,77)$ & $1,09^{c, d, e, f, g}(23,00)$ & $1,38^{\mathrm{b}, \mathrm{c}, \mathrm{d}}(44,55)$ & $3,65^{a, b, c, d, e, f}(9,13)$ & $0,37^{\mathrm{a}, \mathrm{b}, \mathrm{c}, \mathrm{d}, \mathrm{e}, \mathrm{g}, \mathrm{gh}}(18,79)$ \\
\hline $\mathrm{J} 1 *$ & Aglomerado & $3,31^{\text {a,b,c,d,e,f }}(20,49)$ & $21,73^{\mathrm{h}, \mathrm{ij}}(9,63)$ & $1,41^{\mathrm{b}, \mathrm{c}}(10,42)$ & $1,03^{\mathrm{c}, \mathrm{de}, \mathrm{e}}(11,00)$ & $2,32^{\mathrm{j}}(8,16)$ & $0,29^{\mathrm{f}, \mathrm{g}, \mathrm{h}, \mathrm{i}}(32,40)$ \\
\hline $\mathrm{K} 1 *$ & Liofilizado/Gourmet & $4,48^{\mathrm{a}}(4,97)$ & $43,70^{\mathrm{a}}(5,65)$ & $2,15^{\mathrm{a}}(5,23)$ & $2,37^{\mathrm{a}}(16,94)$ & $2,85^{g, h, i}(2,06)$ & $0,25^{\mathrm{i}}(5,96)$ \\
\hline $\mathrm{L} 1 * * *$ & Aglomerado & $3,32^{a, b, c, d, e, f}(1,93)$ & $22,85^{\mathrm{h}, \mathrm{i}, \mathrm{j}}(0,37)$ & $0,86^{\mathrm{f}, \mathrm{g}, \mathrm{h}, \mathrm{i}, \mathrm{j}, \mathrm{k}, \mathrm{l,m}}(0,58)$ & $0,93^{\mathrm{c}, \mathrm{d}, \mathrm{e}}(0,30)$ & $3,50^{a, b, c, d, e, f}(0,30)$ & $0,44^{\mathrm{a}, \mathrm{b}, \mathrm{c}}(1,89)$ \\
\hline $\mathrm{M} 1 * *$ & Aglomerado & $4,07^{\mathrm{a}, \mathrm{b}, \mathrm{c}, \mathrm{d}, \mathrm{e}, \mathrm{f}}(15,65)$ & $25,12^{\mathrm{e}, \mathrm{f,g}, \mathrm{h}}(13,37)$ & $0,81^{\mathrm{f}, \mathrm{g}, \mathrm{h}, \mathrm{i}, \mathrm{j}, \mathrm{k}, \mathrm{l}, \mathrm{m}}(33,79)$ & $0,98^{\mathrm{c}, \mathrm{d}, \mathrm{e}}(48,94)$ & $3,93^{\mathrm{a}, \mathrm{b}, \mathrm{c}, \mathrm{d}, \mathrm{e}}(0,88)$ & $0,39^{\mathrm{a}, \mathrm{b}, \mathrm{c}, \mathrm{d}, \mathrm{f}, \mathrm{f}, \mathrm{g}}(3,49)$ \\
\hline $\mathrm{N} 1 * * *$ & Aglomerado & $3,51^{\text {a,b,c,c,d,e,f }}(1,64)$ & $22,32^{\mathrm{h}, \mathrm{i}, \mathrm{j}}(0,92)$ & 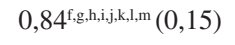 & $1,03^{\mathrm{c}, \mathrm{d}, \mathrm{e}}(0,19)$ & $3,33^{\mathrm{de}, \mathrm{ef}, \mathrm{g}, \mathrm{h}}(0,08)$ & $0,43^{\mathrm{a}, \mathrm{b}, \mathrm{c}}(3,57)$ \\
\hline $\mathrm{P} 1 * * *$ & Aglomerado & $4,09^{a, b, c, d, e, f}(0,78)$ & $21,73^{\mathrm{h}, \mathrm{ij}}(5,20)$ & $1,32^{\mathrm{b}, \mathrm{c}, \mathrm{d}, \mathrm{e}}(0,05)$ & $2,09^{\mathrm{a}, \mathrm{b}}(0,00)$ & $3,22^{\mathrm{f}, \mathrm{g}, \mathrm{h}, \mathrm{i}}(0,17)$ & $0,26^{\mathrm{h}, \mathrm{i}}(1,97)$ \\
\hline Q1* & Aglomerado & $3,47^{\text {a,b,c,d,e, }, \mathrm{f}}(6,71)$ & $21,73 \mathrm{~h}, \mathrm{i}, \mathrm{j}(4,81)$ & $0,73^{\mathrm{h}, \mathrm{i}, \mathrm{j}, \mathrm{k}, \mathrm{l,m}}(0,89)$ & $0,76^{\mathrm{d}, \mathrm{e}}(1,68)$ & $3,98^{\mathrm{a}, \mathrm{b}}(0,83)$ & $0,34^{c, d, e, f, g, h, i, i}(2,83)$ \\
\hline Valores & & 3,64 & 25,58 & 1,00 & 1,20 & 3,51 & 0,36 \\
\hline
\end{tabular}

* Média de 3 lotes e entre parêntesis o CV\% entre lotes, ** média de 2 lotes e entre parêntesis o CV\% entre lotes, *** média de duplicata e entre parêntesis o CV\% analítico. ${ }^{1}$ Valores médios de duplicata (trigonelina, 5-ACQ, cafeína) e triplicata (umidade, luminosidade, melanoidinas) analítica. ${ }^{2}$ Letra maiúscula referente ao Fabricante (Marca), seguido de número referente ao produto. ${ }^{3}$ Letras diferentes na mesma coluna indicam diferença significativa (Tukey, $\mathrm{p} \leq 0,05$ ).

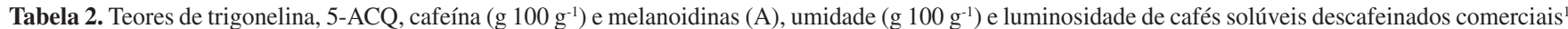

\begin{tabular}{cccccccc}
\hline Produto $^{2}$ & Denominação & Umidade $^{3}$ & Luminosidade $^{3}$ & Trigonelina $^{3}$ & 5-ACQ $^{3}$ & Cafeína $^{3}$ & Melanoidinas $^{3}$ \\
\hline A5** & Aglomerado & $4,08^{\mathrm{b}}(3,35)$ & $22,77^{\mathrm{b}}(3,89)$ & $1,10^{\mathrm{b}}(4,81)$ & $1,18^{\mathrm{b}}(2,38)$ & $0,06^{\mathrm{b}}(8,48)$ & $0,37^{\mathrm{a}}(5,26)$ \\
C6* & Aglomerado & $4,18^{\mathrm{b}}(2,58)$ & $26,01^{\mathrm{a}}(0,56)$ & $1,36^{\mathrm{a}, \mathrm{b}}(5,26)$ & $1,68^{\mathrm{b}}(10,03)$ & $0,12^{\mathrm{b}}(6,81)$ & $0,31^{\mathrm{a}}(6,20)$ \\
D3 $^{*}$ & Aglomerado & $3,39^{\mathrm{b}}(3,09)$ & $26,88^{\mathrm{a}}(3,26)$ & $1,47^{\mathrm{a}, \mathrm{b}}(11,74)$ & $1,73^{\mathrm{a}, \mathrm{b}}(7,21)$ & $0,08^{\mathrm{b}}(18,96)$ & $0,32^{\mathrm{a}}(8,07)$ \\
E3* & Aglomerado & $4,22^{\mathrm{b}}(4,48)$ & $21,70^{\mathrm{b}}(4,26)$ & $1,29^{\mathrm{b}}(19,14)$ & $1,37^{\mathrm{b}}(26,71)$ & $0,11^{\mathrm{b}}(44,94)$ & $0,36^{\mathrm{a}}(9,92)$ \\
G2 $^{* * *}$ & Pó & $6,67^{\mathrm{a}}(1,88)$ & $26,60^{\mathrm{a}}(1,83)$ & $1,85^{\mathrm{a}}(0,02)$ & $2,43^{\mathrm{a}}(0,10)$ & $0,24^{\mathrm{a}}(0,47)$ & $0,33^{\mathrm{a}}(3,14)$ \\
O1*** & Aglomerado & $3,79^{\mathrm{b}}(2,42)$ & $21,65^{\mathrm{b}}(2,58)$ & $1,40^{\mathrm{a}, \mathrm{b}}(0,03)$ & $1,72^{\mathrm{b}}(0,16)$ & $0,09^{\mathrm{b}}(0,06)$ & $0,32^{\mathrm{a}}(3,41)$ \\
\hline Valores Médios & & 4,52 & 24,27 & 1,41 & 1,68 & 0,12 & 0,33 \\
\hline
\end{tabular}

* Média de 3 lotes e entre parêntesis o CV\% entre lotes, ** média de 2 lotes e entre parêntesis o CV\% entre lotes, *** média de duplicata e entre parêntesis o CV\% analítico. ${ }^{1}$ Valores médios de duplicata (trigonelina, 5-ACQ, cafeína) e triplicata (umidade, luminosidade, melanoidinas) analítica. ${ }^{2}$ Letra maiúscula referente ao Fabricante (Marca), seguido de número referente ao produto. ${ }^{3}$ Letras diferentes na mesma coluna indicam diferença significativa (Tukey, $\mathrm{p} \leq 0,05$ ).

produto em base seca para cafés regulares e na faixa de 1,10 a $1,85 \mathrm{~g}$ $100 \mathrm{~g}^{-1}$ para cafés descafeinados, observando-se diferença significativa entre as marcas nas duas categorias de produtos (Tabelas 1 e 2). Essa variabilidade poderia ser atribuída a diferenças na matéria-prima, mas provavelmente está mais associada à diversidade nos processos empregados de torra e extração. Na literatura são relatados teores de 0,35 a $1,69 \mathrm{~g}$ de trigonelina $100 \mathrm{~g}^{-1}$ para cafés solúveis regulares e

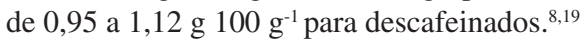

Para 5-ACQ observou-se ainda maior variação: 0,38 a 2,66 g

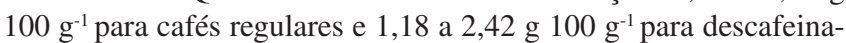
dos (Tabelas 1 e 2). Para cafés solúveis comerciais, foram descritos

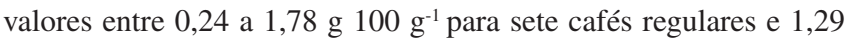

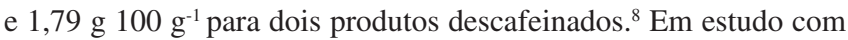
cafés solúveis com vários graus de torra e de diferentes espécies que foram submetidos a diferentes processos de extração, relatou-se que os teores de 5-ACQ variaram de 0,21 a 4,24 g $100 \mathrm{~g}^{-1}$, sendo que os 

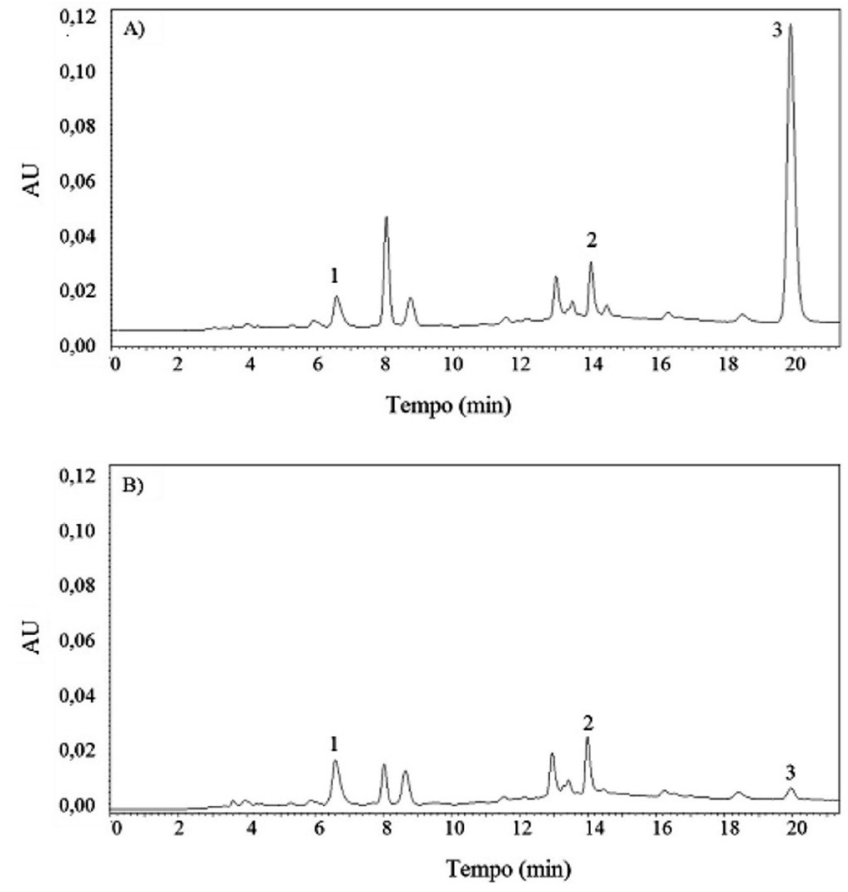

Figura 1. Cromatograma típico de café solúvel regular (A) e descafeinado (B) com detecção a $272 \mathrm{~nm}$. Picos: trigonelina (1); 5-ACQ (2) e cafeína (3)

maiores valores foram observados para produtos feitos com café robusta, torra clara e extração empregando temperaturas mais baixas. ${ }^{10}$

Para cafeína, os teores nos produtos regulares apresentaram menor variabilidade, de 2,32 a 4,08 g $100 \mathrm{~g}^{-1}$ (Tabela 1). Essa variação pode ser mais facilmente atribuída à matéria-prima uma vez que a cafeína, diferentemente da trigonelina e 5-ACQ, é termicamente estável, ${ }^{25}$ assim os processos de torra, extração e secagem devem ter menor influência no teor final de cafeína no produto. Para cafés solúveis comerciais foi relatada concentração na faixa de 1,6 a 3,2 g de cafeína $100 \mathrm{~g}^{-1} .{ }^{8}$ Maior variabilidade $\left(2,84\right.$ a 5,82 g de cafeína $\left.100 \mathrm{~g}^{-1}\right)$ foi descrita em estudo com produtos formulados com grande variação nas condições de matéria-prima (espécie de café) e diversidade nas condições de processo (grau de torra e extração). ${ }^{10}$

Os produtos descafeinados apresentaram concentração de 0,06 a 0,24 g de cafeína $100 \mathrm{~g}^{-1}$, sendo que o café $\mathrm{G} 2$ se diferenciou pelo teor mais alto (Tabela 2). Segundo a legislação brasileira que regulamenta características mínimas de qualidade de produtos de café, o teor máximo de cafeína em produtos solúveis descafeinados é de $0,3 \%$, portanto, todas as amostras avaliadas, considerando-se o valor médio entre lotes, se encontram dentro do preconizado pela legislação. ${ }^{26} \mathrm{Em}$ estudo com oito cafés solúveis descafeinados, uma faixa de valores mais ampla (de 0,058 a $0,4 \mathrm{~g}$ de cafeína $100 \mathrm{~g}^{-1}$ ) foi citada. ${ }^{17}$

Nas amostras avaliadas os produtos descafeinados apresentavam concentração de trigonelina e 5-ACQ superiores à dos cafés regulares quando se comparou produtos da mesma marca. Observou-se

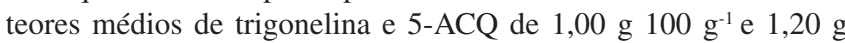
$100 \mathrm{~g}^{-1}$, respectivamente, para produtos regulares contra $1,14 \mathrm{~g} 100$

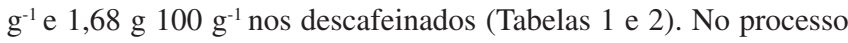
de descafeinação, a cafeína é reduzida na composição do produto, portanto, as porcentagens dos demais compostos são modificadas no extrato seco, tendendo a aumentar proporcionalmente seus teores desde que não tenham sido eluídos simultaneamente com a cafeína na descafeinação. Deve-se considerar ainda que, como a descafeinação altera a estrutura da parede dos grãos aumentando a porosidade, produtos descafeinados podem ser submetidos a processos menos drásticos de torra. ${ }^{5} \mathrm{Na}$ literatura são descritas perdas expressivas de ácidos clorogênicos nos grãos verdes de cafés arábica e robusta na descafeinação com diclorometano, no entanto, valores similares de 5-ACQ foram obtidos para café arábica torrado e moído regular e descafeinado em torra escura. ${ }^{12,27}$ Comparando-se produtos regulares e descafeinados, teores similares de trigonelina foram reportados para cafés arábica torrados (de grau médio a escuro), observando-se maiores teores do mesmo composto no café robusta descafeinado cru e em todos os graus de torra. ${ }^{12}$

Para melanoidinas, o único dos componentes bioativos estudados que foi formado no processo de torra, observou-se valores de absorvância de 0,253 e 0,476 para cafés regulares (Tabela 1) e valor médio de 0,330 para descafeinados (Tabela 2), onde não houve diferença entre produtos descafeinados. Em estudo com cafés solúveis, relatou-se que os teores de melanoidinas variaram com a espécie de café e processo de extração, mas foram mais afetados pelo grau de torra. ${ }^{10} \mathrm{~A}$ maior variabilidade na melanoidinas observada para os cafés regulares poderia assim ser atribuída a uma maior variação no grau de torra nessa categoria de produto, já indicada pelas grandes variações no L* e nos componentes termolábeis $(4,5$ e 7 vezes, respectivamente, para teores de trigonelina e 5-ACQ) (Tabela 1).

Para cafés torrados e moídos foi citada correlação entre teor de melanoidinas e $\mathrm{L}^{*}$, onde produtos com menor grau de torra possuíam menor valor de $\mathrm{L}^{*}$ e melanoidinas para cafés torrados e moídos comerciais. ${ }^{21}$

No entanto, não foi observada correlação entre a luminosidade (L*) medida no produto seco e a presença de compostos escuros (Tabelas 1 e 2) para os cafés solúveis estudados. No café solúvel, a luminosidade é dependente tanto do grau de torra quanto das condições do processo de secagem. ${ }^{5,28}$ Em estudo com café solúvel em que os autores trabalharam com processo de secagem padronizado para todas as amostras, consideraram como melanoidinas a fração de massa molecular entre 12 e $14 \mathrm{kDa}$, foram reportados teores mais altos de melanoidinas nos cafés solúveis mais torrados. Os autores observaram, porém, que mesmo com a padronização no preparo das amostras, incluindo as mesmas condições de secagem, em um dos tipos de extração estudados, as amostras de torra clara apresentaram maior teor de melanoidinas do que cafés com torra média. ${ }^{10}$

Nossos resultados mostraram que, para um produto solúvel comercial onde não se tem informação do grau de torra, a avaliação de intensidade do processo de torra não deve ser baseada somente em parâmetros de cor, mas também na avaliação dos componentes que podem ser formados e perdidos no processo.

Não se observou também correlação entre o tipo de secagem empregado na fabricação do café solúvel e o balanço de componentes bioativos na composição. Para os produtos liofilizados (B2, C2, e K1) observaram-se altos valores de trigonelina e 5-ACQ e baixos valores de cafeína, quando comparados aos produtos aglomerados e em pó e a média geral para cafés solúveis regulares (Tabela 1). No entanto, parte dessa variação poderia ser atribuída à matriz de café utilizada, uma vez que os cafés aglomerados e em pó, que não tinham especificação dos fabricantes, devem ser produtos de blends das espécies robusta e arábica e, os liofilizados foram descritos como Gourmet, indicando o uso de café arábica. Cabe ressaltar também que é recomendado o emprego de torra menos intensa (de médio claro a quase médio) para cafés Gourmet.$^{29} \mathrm{O}$ café K1, por exemplo, apresentou maior teor dos componentes termolábeis (trigonelina e 5-ACQ) e menor presença de compostos escuros, indicando menor grau de torra (Tabela 1). Assim, os dados de composição indicam que as diferenças nos produtos liofilizados devem ser mais devidas ao emprego de matéria-prima e torra adequadas aos produtos Gourmet do que ao processo de secagem em si.

Com relação às variações em lotes dos mesmos produtos $(2$ ou 3 lotes), observou-se para cafés regulares que as diferenças na 
composição foram mais expressivas para os compostos formados ou perdidos no processo de torra como trigonelina (CV de até $23 \%$ ), 5-ACQ (até 44,55\%) e melanoidinas (até 32,40\%). Para cafeína, cuja variação poderia ser mais atribuída à diversidade de matéria-prima, os CVs foram inferiores a 9,13\% (Tabela 1).

Para cafés solúveis descafeinados, além da variabilidade observada para trigonelina (CV até 19,14\%), 5-ACQ (até 26,71\%) e melanoidinas (até 9,92\%), observou-se também variação para cafeína (até 44,94\%) atribuída à diversidade entre os lotes dos grãos obtidos do processo de descafeinação (Tabela 2). Em estudo com cafés descafeinados torrados e moídos e solúveis foi relatada grande diferença de teor de cafeína entre diferentes lotes da mesma marca, reportando $\mathrm{CV}$ na faixa de 5 a $25 \%$ para cafés solúveis e 6 a $111 \%$ para os torrados. ${ }^{17}$

\section{CONCLUSÃO}

Apesar da diversidade de matéria-prima e dos processos empregados na produção, todos os produtos comerciais analisados apresentaram teores expressivos de compostos bioativos, porém com grande variação (CVs de até $45 \%$ ) entre os lotes de um mesmo produto. Em geral, os cafés Gourmet e os descafeinados apresentavam maior teor de trigonelina e 5-ACQ e menos cafeína e melanoidinas que os produtos regulares.

\section{AGRADECIMENTOS}

Ao CNPq pelas bolsas de Iniciação Científica, Mestrado e Produtividade em Pesquisa.

\section{REFERÊNCIAS}

1. http://www.abic.com.br, acessada em Fevereiro 2013.

2. http://www.agricultura.gov.br, acessada em Fevereiro 2013.

3. http://www.ceplac.gov.br, acessada em Fevereiro 2013.

4. Fernandes, S. M.; Pereira, R. G. F. A.; Pinto, N. A. V. D.; Nery, M. C.; Pádua, R. F. M.; Ciênc. Agrotec. 2003, 27, 1076.

5. Clarke, R. J.; Vitzthum, O. G.; Coffee: Recent Developments, Blackwell Science: London, 2001.

6. Alves, R. C.; Casal, S.; Oliveira, B.; Quim. Nova 2009, 32, 2169.

7. Brasil; Portaria $n^{\circ} 130$, de 19 de fevereiro 1999, www.anvisa.gov.br/ legis/portarias/ 130_99.htm, acessada em Fevereiro 2013.

8. Nogueira, M.; Trugo, L. C.; Ciên. Tecnol. Aliment. 2003, 23, 296.
9. Dias, R. C. E.; Dissertação de Mestrado, Universidade Estadual de Londrina, Brasil, 2005; Ky, C. L.; Louarn, J.; Dussert, S.; Guyot, B.; Hamon, S.; Noirot, M.; Food Chem. 2001, 75, 223.

10. Vignoli, J. A.; Bassoli, D. G.; Benassi, M. T.; Food Chem. 2011, 124, 863.

11. Morais, S. A. L.; Aquino, F. J. T.; Nascimento, P. M. N.; Nascimento, E. A.; Chang, R.; Quim. Nova 2009, 32, 327.

12. Toci, A.; Farah, A.; Trugo, L. C.; Quim. Nova 2006, 29, 965.

13. Esquivel, P.; Jiménez, V. M.; Food Res. Int. 2012, 46, 488.

14. Higdon, J. V.; Frei, B.; Crit. Rev. Food Sci. Nutr. 2006, 46, 101.

15. De Maria, C. A. B.; Moreira, R. F. A.; Quim. Nova 2011, 34, 1739.

16. Delgado-Andrade, C.; Rufián-Henares, J. A.; Morales F. J.; J. Agric. Food Chem. 2005, 53, 7832.

17. Meinhart, A. D.; Bizzotto, C. S.; Ballus, C. A.; Prado, M. A.; Bruns, R. E.; Teixeira Filho, J.; Godoy, H. T.; Food Chem. 2010, 120, 1155.

18. Perrone, D.; Donangelo, C. M.; Farah, A.; Food Chem. 2008, 110, 1030 ; Trugo, L. C.; De Maria, C. A. B.; Werneck, C. C.; Food Chem. 1991, 42, 81; Trugo, L. C.; Macrae, R.; Analyst 1984, 109, 263.

19. Trugo, L. C.; Macrae, R.; Dick, J.; J. Sci. Food Agric. 1983, 34, 300.

20. López-Galilea, I.; Paz De Peňa, I.; Cid, C.; J. Agric. Food Chem. 2007, 55,6110 .

21. Almeida, M. B.; Benassi, M.T.; Semina Ciênc. Agrar. 2011, 32, 1893.

22. Bekedam, E. K.; Schols, H. A.; van Boekel, M. A. J. S.; Smit, G.; J. Agric. Food Chem. 2006, 54, 7658; Charurin, P.; Ames, J. M.; Del Castillo, M. D.; J. Agric. Food Chem. 2002, 50, 3751; Del Castillo, M. D.; Ames, J. M.; Gordon, M. H.; J. Agric. Food Chem. 2002, 50, 3698.

23. Alves, S. T.; Dias, R. C. E.; Benassi, M. T.; Scholz, M. B. S.; Quim. Nova 2006, 29, 1164.

24. Statsoft 7.1; Statistic for Windows: Computer program manual; Tulsa, 2006.

25. De Souza, R. M. N.; Canuto, G. A. B.; Dias, R. C. E.; Benassi, M. T.; Quim. Nova 2010, 33, 885; Monteiro, M. C.; Trugo, L. C.; Quim. Nova 2005, 28, 637.

26. Brasil; Resolução $M S-R D C n^{\circ} 277$, de 22 de setembro de 2005, www. bvsms.saude.gov. br/bvs/saudelegis/anvisa/2005/res0277_22_09_2005. html, acessada em Fevereiro 2013.

27. Farah, A.; De Paulis, T.; Moreira, D. P.; Trugo, L. C.; Martin, P. R.; J. Agric. Food Chem. 2006, 54, 374.

28. http://www.niro.com/niro/cmsdoc.nsf/WebDoc/webb7mggv8, acessada em Setembro 2012

29. São Paulo; Resolução $S A A n^{\circ} 31$, de 22 de junho de 2007, www.abic. com.br/publique/ media/CONS_leg_resolucaoSAA 31.pdf, acessada em Fevereiro 2013. 